\title{
ANGLE-RESOLVED PHOTOELECTRON SPECTROSCOPY ON CdTe(100) $\mathrm{c}(2 \times 2)$
}

\author{
K.-U. GaWlik, J. Brügmann, S. HaRM, R. Manzke, M. Skibowski \\ Institut für Experimentalphysik, Universität Kiel \\ Olshausenstr. 40-60, 24098 Kiel, Germany \\ B.J. Kowalski and B.A. Orlowski \\ Institute of Physics, Polish Academy of Sciences \\ Al. Lotników 32/46, 02-668 Warszawa, Poland
}

(Received June 1, 1993)

\begin{abstract}
The $\mathrm{CdTe}(100) c(2 \times 2)$ surface prepared by ion bombardment and annealing was investigated by angle-resolved photoemission spectroscopy using synchrotron radiation with photon energies between $8.5 \mathrm{eV}$ and $32 \mathrm{eV}$. The bulk band structure was determined along the $\Gamma \Delta X$-direction normal to the surface measuring energy-distribution curves. The results are compared with a theoretical valence band structure assuming free-electron-like final states in connection with $k$-conservation. For further comparison we calculated the final states by the pseudopotential method and analyzed the results in form of structure plots. In this way, most of the dispersing maxima in the normal emission spectra can be explained. For this mode of photoelectron spectroscopy the resulting dispersion must be due to the component of the Bloch wave vector normal to the surface, $k_{\perp}$, keeping surface related structures at a fixed binding energy position $E_{\mathrm{b}}$. The lack of dispersion for five structures along $\Gamma \Delta X$ gives experimental evidence for their surface origin.

PACS numbers: 73.20.At, 79.60.Bm
\end{abstract}

\section{Introduction}

With its increasing technological importance, especially concerning the fabrication of optoelectronical devices, $\mathrm{CdTe}$ has also become an interesting material in basic research. Information about the electronic properties of this prototype II-VI compound semiconductor gives the possibility to develop reliable models of the clean surface or of interfaces between semiconductors and metals or between different semiconductors. Because of the simple preparation of smooth and clean surfaces by cleaving bulk crystals under ultrahigh-vacuum (UHV) conditions the 
(110) surface of CdTe is the best experimentally studied plane [1-4]. Surface atoms react only by relaxation instead of more complicated surface reconstructions. This is the reason for the fact that the (110) surface is also the best theoretically investigated plane of CdTe and a surface model has been established [5].

Other low-indexed crystal planes like the (100) and (111) surfaces have also become subject of band structure investigations [6-8] by angle-resolved photoelectron spectroscopy (ARPES). Recently we reported results of ARPES studies of an unreconstructed $\mathrm{CdTe}(100)$ surface prepared by ion bombardment and annealing [9]. Using the same technique it is possible to prepare a $c(2 \times 2)$ reconstructed (100) surface which Wu et al. [10] prepared by molecular beam epitaxy (MBE) and found it to be Te terminated.

In this work we present the first experiments on the $\mathrm{CdTe}(100) \mathrm{c}(2 \times 2)$ surface with the aim to investigate the experimental band structure from normal emission energy-distribution curves (EDC's). In a detailed analysis the experimental results are compared with theoretical bulk band structures applying structure plots, a method described in detail by Olde et al. [11]. This technique is an excellent tool to compare theoretical predictions with experimental data. We explain the binding energies and dispersion of the main maxima by transitions from the valence bands into backfolded free-electron-like final states using a proper value for the inner potential. To refine these results we calculated the unoccupied states by the empirical pseudopotential method (EPM). Using structure plots with these final states we obtain deeper insight into the accuracy of the valence and conduction band calculations. Especially the maxima with no significant dispersion are not found in the structure plots and must be attributed to high density of states effects and moreover surface effects.

\section{Experimental}

The angle-resolved photoemission spectra were taken with synchrotron radiation in a photon energy range of $8.5 \mathrm{eV} \leq h \nu \leq 32 \mathrm{eV}$ supplied by the storage ring DORIS III at HASYLAB in Hamburg, Germany, with an energy resolution of $130 \mathrm{meV}$. The electrons were detected by a spherical analyzer mounted on a two-axes goniometer. The angle-resolution was better than $0.5^{\circ}$.

The $\mathrm{CdTe}(100)$ wafers with dimensions of $5 \times 5 \times 0.5 \mathrm{~mm}^{3}$ were cut from CdTe single crystals which were grown by the modified Bridgman method in the Institute of Physics, Polish Academy of Sciences, and oriented by standard X-ray diffraction. All samples investigated here were cleaned by $500 \mathrm{eV}$ argon ion bombardment for 15 minutes and then annealed at about $260^{\circ} \mathrm{C}$ for 2 hours. The surface structure was characterized by means of low-energy electron diffraction (LEED) and reflection high-energy electron diffraction (RHEED) in an ultrahigh-vacuum (base pressure in the low $10^{-10} \mathrm{mbar}$ range) preparation system. From both methods we obtained a reconstructed $\mathrm{CdTe}(100) \mathrm{c}(2 \times 2)$ surface by the preparation procedure given above. 


\section{Results and discussion}

Angle-resolved photoemission spectra of $\operatorname{CdTe}(100) \mathrm{c}(2 \times 2)$ taken in normal emission along the high symmetry direction $\Gamma \Delta X$ of the bulk Brillouin zone within a photon energy range between $8.5 \mathrm{eV}$ and $32 \mathrm{eV}$ are shown in Fig. 1. One

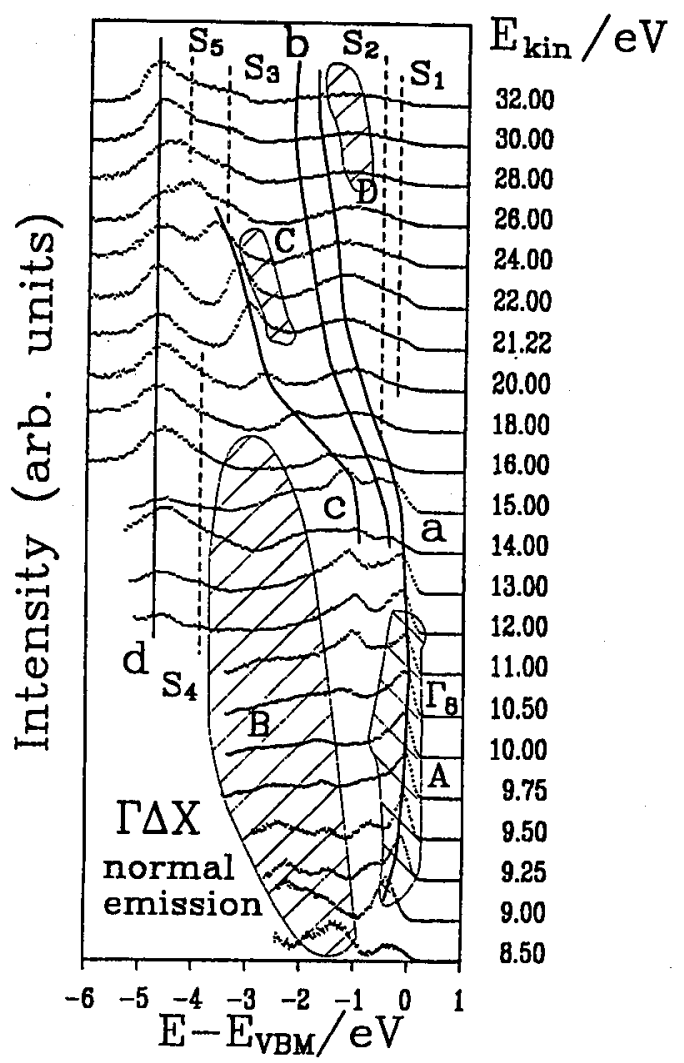

Fig. 1. Normal-emission energy-distribution curves of $\operatorname{CdTe}(100) c(2 \times 2)$. The non-dispersing structures $S_{1}-S_{5}$ are attributed to transitions from real surface states or resonances whereas $d$ is due to high density of states effects at the critical point $X_{6}$. The dispersing maxima $a-c$ are used to determine the inner potential and those lying inside the regions $A-D$ will be discussed particularly by structure plots. The peak positions related to the VBM of all spectra are determined by a Gaussian fit.

can follow the structures $a, b$, and $c$ in a wide range of photon energies. These features, used later to determine the inner potential of this crystal surface, show clear dispersion and thus are attributed to transitions from bulk states. Line $a$ labels the first bulk emission maxima at highest kinetic energy or vice versa at low binding energy of each spectrum. These maxima are attributed to direct transitions from the $\Gamma_{8} X_{7}$ valence band into a final state band. One can continuously 
follow the dispersion of the first structure with decreasing photon energy down to $10.5 \mathrm{eV}$ moving towards lower binding energy where we detect direct transitions from the valence band maximum $\Gamma_{8}(\mathrm{VBM})$. Further reducing the photon energy, this structure disperses to higher binding energy again. This behaviour rules out a final state band splitting as postulated by Niles and Höchst [6] who investigated the $\mathrm{CdTe}(100)(2 \times 1)$ surface by angle-resolved photoemission in normal emission with larger stepsize concerning the photon energy in the $10 \mathrm{eV}$ range. These authors could not resolve this slight dispersion to higher binding energy in their EDC's. $S_{1}-S_{5}$ seem to be true surface states or resonances while $d$ is mainly caused by bulk effects. Additionally we show four labelled regions $(A-D)$ which will be discussed below. The manifold of maxima observed in these areas are not explicitly labelled but are also extracted by Gaussian fits and taken into consideration in the structure plots.

We now turn to the method of structure plots previously used by Williams et al. [12] and Olde et al. [11] for the interpretation of the (110) and (001) surfaces of III-V semiconductors, respectively. This method has proved to be very useful for the detailed analysis of normal emission spectra. The basic idea of this technique is to choose a pair of bands, a valence band $E_{\mathrm{b}, \mathrm{v}}\left(k_{\perp}\right)$ and conduction band $E_{\mathrm{b}, \mathrm{c}}\left(k_{\perp}\right)$, where $k_{\perp}$ is the wave vector component of electrons perpendicular to the surface. The procedure is to determine the transition energies between the selected valence and conduction band for $k$-conserving transitions for all $k_{\perp}$ values of the Brillouin zone, i.e., an excitation is possible for $k_{\perp 0}$ for a photon energy $h \nu_{0}$ at the initial state $E_{\mathrm{b}, \mathrm{v}}\left(k_{\perp 0}\right)$. The resulting pairs $E_{\mathrm{b}, \mathrm{v}}\left(k_{\perp 0}\right)$ and $h \nu_{0}$ are plotted into an $E_{\mathrm{b}}(h \nu)$ diagram. Proceeding in the same way for all other pairs of occupied and unoccupied bands a collection of curves like that in Fig. $2 a$ and $2 b$, called structure plot, is obtained. These structure plots are directly comparable with experimental data. Chelikowsky and Cohen [13] performed nonlocal empirical pseudopotential calculations for $\mathrm{CdTe}$ which we used as valence bands $E_{\mathrm{b}, \mathrm{v}}\left(k_{\perp}\right)$ for the structure plots of Fig. 2.

Assuming first free-electron-like final states and direct transitions the unoccupied states are described by the well-known equation

$$
E_{\mathrm{b}, \mathrm{f}}\left(k_{\perp}\right)=\frac{\hbar^{2}}{2 m}\left[\left(k_{\perp}+G_{\perp}\right)^{2}+g_{\|}^{2}\right]-\left|E_{0}\right|
$$

with $\left|E_{0}\right|=\left|V_{0}\right|-U_{\text {th }}$, where $\left|E_{0}\right|$ is the inner potential related to the VBM and $G_{\perp}$ the normal component of a bulk reciprocal lattice vector $\boldsymbol{G}=G_{\perp}+G_{\|}$. The vector $g_{\|}$is generally a linear combination of the parallel component of the reciprocal bulk lattice vector $\boldsymbol{G}$ and a reciprocal lattice vector of the ideal or reconstructed surface. The inner potential $\left|V_{0}\right|$ related to the vacuum level differs from $\left|E_{0}\right|$ by the photoelectron threshold $U_{\text {th }}$. Figure 2a shows the structure plot for the free-electron-like final state approximation using the 125 shortest reciprocal bulk lattice vectors. Many of these parabolas are degenerate along the $\Gamma \Delta X$ direction which reduces the number of curves in the structure plot. $\left|V_{0}\right|=9.5 \mathrm{eV}$ was determined by finding the best agreement between the experimental $E_{\mathrm{b}}(h \nu)$ positions of the emission maxima $a, b$, and $c$ with photon energy larger than $11 \mathrm{eV}$ (full symbols in Fig. 2) and the structure plot using only the main branch of the free-electron parabola normal to the surface as final state in the structure plot, 

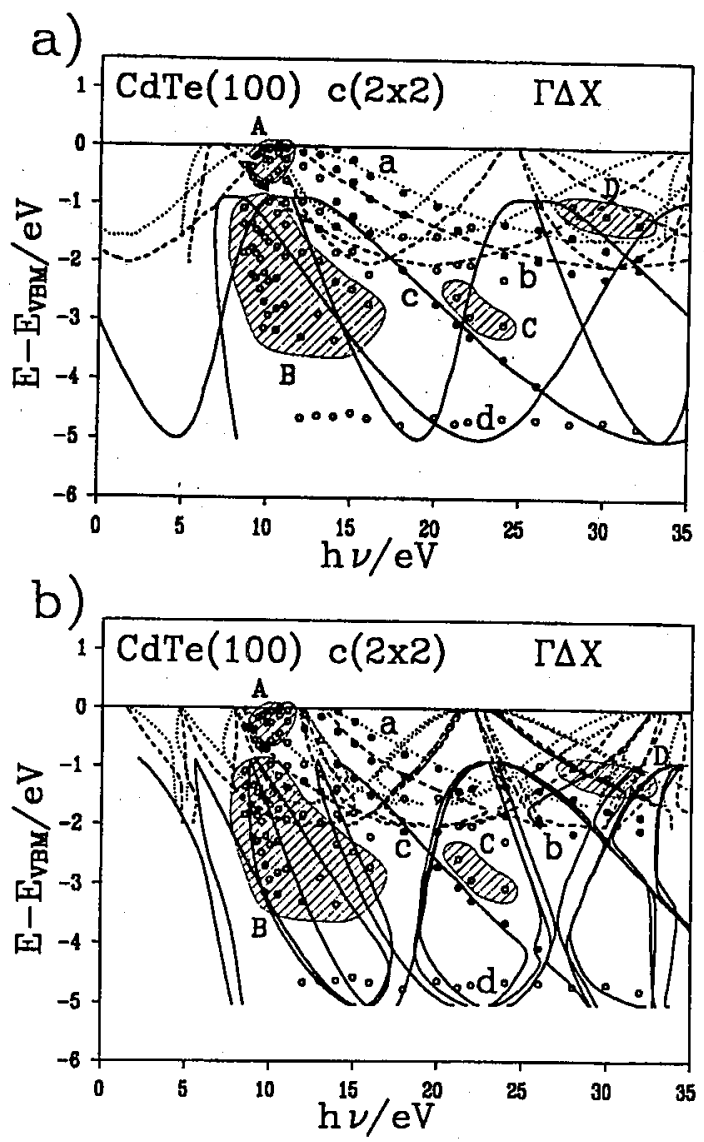

Fig. 2. Structure plots for free-electron-like final states ((a) top) and conduction bands calculated by means of a nonlocal pseudopotential method ((b) bottom). Only the maxima corresponding to the experimental features $a, b$, and $c$ of Fig. 1 which are used to determine the inner potential are marked by full circles. The lines represent theoretically predicted direct electron transitions from states with a binding energy $E-E_{\mathrm{VBM}}$ via photons with energy $h \nu$ into conduction bands. Transitions from the $\Gamma_{8} X_{7}, \Gamma_{8} X_{6}$, and $\Gamma_{7} X_{6}$ valence bands are plotted as dotted, dashed, and solid lines, respectively. Differences concerning these two types of structure plots are discussed for the regions $A-D$ (see text). We neglected the surface related states in this picture.

e.g., $g_{\|}=0 \AA^{-1}$ and $G_{\perp}=4 \pi / a$ where $a=6.481 \AA$. We found good agreement between theory and experiment in the region $A$ although these maxima were not taken into consideration to determine the inner potential $\left|V_{0}\right|$. This result is not directly obvious because larger deviations of the free-electron parabola have to be expected for lower binding energy of unoccupied states. Especially, near the 
centre of the Brillouin zone, the periodic crystal potential can cause additional band splitting. This deviation becomes important for the structures in region $B$ where we find only few lines which do not fit the experimental results adequately concerning energy position and inclination. $U_{\mathrm{th}}=5.61 \mathrm{eV}$ was experimentally determined thus leading to $\left|E_{0}\right|=3.89 \mathrm{eV}$ which differs from the value $\left|E_{0}\right|=4.5 \mathrm{eV}$ reported by Niles and Höchst [6]. Furthermore, only the experimental structures with clear dispersion along the $\Gamma \Delta X$ direction are shown in Fig. 2. An exception is the weakly dispersing structure $d$ around $-4.6 \mathrm{eV}$. This energy position is close to the critical point $X_{6}$ which has an energy of $-5.05 \mathrm{eV}$ following the calculation of Chelikowsky and Cohen. The high density of bulk states associated with the bulk band edge at $X_{6}$ is expected to contribute to this feature. The structures $S_{1}-S_{5}$ which also reveal no dispersion are far away from critical points and are thus clearly identified as surface states or resonances. The structures $C$ and $D$ cannot be attributed to transitions into free-electron-like final states. All other maxima find a corresponding curve in the structure plot of Fig. 2a which means that these peaks can be consistently explained by combining the free-electron-like final state parabola with the valence band structure of Chelikowsky and Cohen if different branches are considered, e.g., $G_{\|} \neq 0 \AA$ or $G_{\perp} \neq 4 \pi / a$.

One knows from conduction band calculations that the periodic potential of the crystal causes the bands to split and the backfolded parabola to shift in energy differently for each conduction band. In order to improve our simple model with free-electron-like final states we now turn to more realistic unoccupied states. Unfortunately, we were not able to use the conduction bands calculated by Chelikowsky and Cohen because these have been given for the lower binding energy region and not for the unoccupied bands into which the photoemission transitions occur. Therefore we used the parameters determined by Chelikowsky and Cohen and calculated the conduction bands in the frame of this local pseudopotential method. With these final states the structure plot shown in Fig. $2 \mathrm{~b}$ has been recalculated. It is important to mention that there exists now no free fit parameter like $\left|V_{0}\right|$ for the free-electron-like final state approximation. The first observation is that the structures used to determine the inner potential can be explained directly by the more realistic final states. Also the maxima not labelled explicitly can now be described by electron transitions from the valence bands into conduction bands calculated by nonlocal EPM or local EPM, respectively. For these transitions the free-electron parabolas coincide with the EPM results. In addition, we find good agreement between theory and experiment for the structures sampled in area $D$. Another success is that the number of transitions curves has increased in region $B$ although not all experimental points can be attributed to a theoretical curve in this region. The most remarkable point is the large distance of any theoretical curve from the maxima sampled in region $A$. This is much better described by the free-electron-like final states. The reason for this failure is a bandgap in the theoretical conduction bands which excludes transitions with photon energy between $9 \mathrm{eV}$ and $11 \mathrm{eV}$ from initial states of binding energy between $0 \mathrm{eV}$ and $-0.5 \mathrm{eV}$. Including of damping in the band structure calculations can avoid this effect. The energy gaps will be smeared out and the agreement between theory and experiment is clearly improved. Similar to the free-electron-like final states 
the structures $C$ cannot be explained. Taking into account nonlocal corrections and spin-orbit interactions this problem may be overcome by additional splittings of the conduction bands.

\section{Conclusion}

We have performed a detailed study of the electronic band structure of $\mathrm{CdTe}(100) \mathrm{c}(2 \times 2)$ along the high symmetry line $\Gamma \Delta X$ of the bulk Brillouin zone by angle-resolved photoelectron spectroscopy in normal emission. Assuming direct electron transitions most of the dispersing maxima in the EDC's can be explained by free-electron-like final states with an inner potential of $\left|V_{0}\right|=9.5 \mathrm{eV}$. Structure plots have been used to compare experimental results with theoretical predictions. We refined the free-electron parabolas by final states from a pseudopotential conduction band calculation which differs from the parabolas particularly in the region of low photon energy. Additionally, we found six states with no significant dispersion. One lying very close to the $X_{6}$ point is attributed to non $k$-conserving bulk transitions caused by the high density of states at this point. The other five non-dispersing structures $S_{1}-S_{5}$, found far away from critical points, are explained as real surface states or resonances.

\section{Acknowledgment}

This work was supported by the Bundesministerium für Forschung und Technologie (project no. 05 5FKAAB) and the Committee for Scientific Research project no. 204869101.

\section{References}

[1] T.P. Humphreys, G.P. Srivastava, R.H. Williams, Solid State Phys. 19, 1259 (1986).

[2] K.O. Magnusson, S.A. Flodström, Phys. Rev. B 38, 5384 (1988).

[3] H. Qu, P.O. Nilsson, J. Kanski, L. Ilver, Phys. Rev. B 39, 5276 (1990).

[4] C. Janowitz, L. Kipp, R. Manzke, B.A. Orłowski, Surf. Sci. 231, 25 (1990).

[5] Y.R. Wang, C.B. Duke, K.O. Magnusson, S.A. Flodström, Surf. Sci. 205, L760 (1988).

[6] D.W. Niles, H. Höchst, Phys. Rev. B 43, 1492 (1991).

[7] B.A. Orłowski, E. Janik, C. Janowitz, R. Manzke, Acta Phys. Pol. A 79, 303 (1991).

[8] C. Janowitz, R. Manzke, M. Skibowski, B.A. Orłowski, Surf. Sci. 247, 100 (1991).

[9] K.-U. Gawlik, J. Brügmann, S. Harm, C. Janowitz, R. Manzke, M. Skibowski, C.-H. Solterbeck, W. Schattke, B.A. Orłowski, Acta Phys. Pol. A 82, 355 (1992).

[10] Y.S. Wu, C.R. Becker, A. Waag, M.M. Kraus, R.N. Bicknell-Tassius, G. Landwehr, Phys. Rev. B 44, 8904 (1991).

[11] J. Olde, G. Mante, H.-P. Barnscheidt, L. Kipp, J.-C. Kuhr, R. Manzke, M. Skibowski, J. Henk, W. Schattke, Phys. Rev. B 41, 9958 (1990).

[12] G.P. Williams, F. Cerrina, G.J. Lapeyre, J.R. Anderson, R.J. Smith, J. Hermanson, Phys. Rev. B 34, 5548 (1986).

[13] J.R. Chelikowsky, M.L. Cohen, Phys. Rev. B 14, 556 (1976). 